\title{
Early Results of ERAS (Enhanced Recovery After Surgery) Protocol in Orthopaedic Surgery
}

Jaydeep Shah MBBS, M.S (T\&O), MRCSEd, FRCSEd (T\&O).- 1, Keshav Singhal MBBS, M.S (T\&O), MCh (Ortho), FRCS.2

1 Associate Specialist, Trauma and Orthopaedics, Princess of Wales Hospital, Bridgend

2 Consultant Orthopaedic Surgeon, Princess of Wales Hospital, Bridgend.

Keshav.singhal@gmail.com

Key words

Early recovery, hip replacement, knee replacement,

Cite as: Shah, J., Singhal, K. (2012) Early results of ERAS (Enhanced recovery after surgery) protocol in Orthopaedic Surgery. The Physician vol1(1);28-32 DOI: 10.38192/1.1.1.09

\section{Abstract}

Background: The concept of fast track (Enhanced Recovery After Surgery, ERAS) was introduced to colorectal surgery in Denmark by Kehlet in 1999 which improved the quality of the care and reduced the length of hospital stay following major colorectal surgery. The same principles of ERAS have been applied to the orthopaedic surgery particularly the hip and knee replacement surgery and fracture neck of femur surgery. It is a relatively new approach in orthopaedics to the preoperative, intraoperative and postoperative care of the patients undergoing surgery.

Methods: We have compared the length of inpatient stay, day of mobilisation, postoperative blood transfusion and adverse outcome for the patients undergoing hip or knee replacement by a single surgeon (KS) between ERAS and NON ERAS patients.

Results: A total of 138 patients underwent hip or knee replacement, hip resurfacing arthroplasty or oxford uni-compartmental arthroplasty between July 2011 and June 2012 with ERAS protocol. In the Non ERAS group, 140 patients underwent hip or knee arthroplasty, resurfacing or oxford uni-compartmental knee replacement in the previous year (July 2010 to June 2011) by the same surgeon.

Average hospital in patient stay for the ERAS patients was 4.12 days with $73.10 \%$ of the patients having an inpatient hospital stay of less than or equal to 5 days. The average hospital in patient stay for the NON ERAS patients was 8.34 days with only $24.08 \%$ of the patients being discharged in less than or equal to 5 days.

Conclusions: Our study shows that the implementation of the ERAS protocol in hip and knee replacement surgery is associated with improved patient experience, faster recovery and shorter hospital in patient stay with no increase in complication.

Introduction 
Total hip and knee replacement are the commonest, most successful and cost effective orthopaedic surgical interventions. They provide reliable pain relief and marked improvement in the function of the patients suffering from Osteoarthritis or inflammatory arthritis of the hip or knee. 10 Typically a patient undergoing hip or knee replacement is admitted day before the planned surgery. Traditionally after the surgery, patient stays in the bed overnight with PCA (Patient controlled analgesia), drain from the operative site and an attempt is made to mobilise out of bed on 1st postoperative day. The average length of stay for a hip or knee replacement is between 5.4 days to 9.1 days for hip replacement, and between 5.3 to 8.1 days for the knee replacement. 7

With the changing times and scrutiny of expenditure along with limitations/cuts on the public spending, more emphasis is put on the efficient use of available resources. At the same time the expectations of the public is increasing with increasing litigations. Reducing the hospital stay should reduce patient morbidity, free up much needed hospital beds and increase the capacity of the hospital. The concept of fast track (Enhanced Recovery After Surgery, ERAS) was introduced to colorectal surgery in Denmark by Kehlet in 1999 which improved the quality of the care and reduced the length of hospital stay following major colorectal surgery.1The same principles of ERAS have been applied to the orthopaedic surgery particularly the hip and knee replacement surgery and fracture neck of femur surgery.

This is a relatively new approach in orthopaedics to the preoperative, intraoperative and postoperative care of the patients undergoing surgery. It is a multi-modality, evidence based approach to improving the quality of patient care after major surgery, with a selected number of individual interventions which, when implemented together, demonstrate a greater impact on the outcomes then when implemented as individual interventions. It is a multidisciplinary approach involving surgeons, anaesthetists, nurses, physiotherapists, dieticians and occupational therapists. 3

ERAS is a part of wales assembly government's 1000 lives Plus campaign. Its aim is to improve the quality of care provided to the patients who undergo major surgery. By improving the quality in care, and reducing the harm it is also assumed that the hospital stay will become more efficient, thereby allowing hospital services to realise the benefits of the programme, through savings in bed days. It has already been shown to benefit patients undergoing colorectal, urological, gynaecological and orthopaedic surgery. 3

\section{The basic principles of the ERAS include:}

1. Ensuring the patient is in the best possible condition for surgery

2. Ensuring the patient has the best possible management during and after his/her operation.

3. Ensuring the patient experiences the best possible rehabilitation, enabling early recovery and discharge from hospital, allowing them to return to their normal activities quicker. 3

The main aspect of ERAS programme in orthopaedic surgery are, day of surgery admission (DOSA), Carbohydrate loading, Anaesthetic management, local infiltration analgesia, avoiding surgical site drain, regular postoperative analgesia and minimising the risk of postoperative nausea and vomiting, early mobilisation. Day of surgery admission (DOSA) has a benefit of reducing the potential for surgical site infection and reducing the post-operative complications. This will also help patients spending less time in the hospital and ultimately provide improved capacity within secondary care. 3 Carbohydrate loading 12 hours and 2-4 hours prior to surgery has also been shown to reduce patient anxiety, reduce preoperative thirst, hunger, and postoperative insulin resistance. It promotes a more anabolic state leading to less post- 
operative nitrogen and protein losses as well as better maintained lean body mass and muscle strength. Nygreet found improvement in insulin resistance with patients given carbohydrate loading and Melis demonstrated it preserved immune function post operatively. 3, 6

Anaesthetic management: The aim is to deliver safe and effective sedation and analgesia to the patient which does not hinder early mobilisation.

For patients receiving general anaesthesia, the protocol is as under.

1. Premed analgesia pregabalin $150 \mathrm{mg}$ one hour preop ( reduce dose to $75 \mathrm{mg}$ if patient aged over 75 years, has renal impairment or low BMI): 27,28,30

2. Minimise/avoid opiates. Use short acting anaesthetics.

3. Intraoperatively patient receives,

4. 1 gm IV paracetamol,

5. a non-steroidal anti-inflammatory,

6. $15-20 \mathrm{mg} / \mathrm{kg}$ Tranexamic acid iv bolus prior to tourniquet.3,25

7. Dexamethasone $8 \mathrm{mg}(28,29,30)$

8. $20 \mathrm{mmol}$ Magnesium Sulphate infusion during surgery (started prior to tourniquet) and to titrate rate to heart rate and blood pressure. 26

9. 1-2 litres of iv fluid

10. Intra/postoperative local infiltration analgesia by the surgeon. 22, 23, 24.

In our institution, we use $30 \mathrm{mls}$ of $5 \%$ chirocaine with $1 \mathrm{ml}$ adrenaline diluted with $70 \mathrm{mls}$ of normal saline.

For patients receiving spinal anaesthesia,

1. Pre-med as above

2. Spinal analgesia aiming at unilateral block wherever possible, and to use minimum dose of anaesthetic to reduce urinary retention and delay in motor function.

Local infiltration analgesia: it gives good post-operative pain control without limiting the mobilisation with evidence showing a decreased use of patient controlled analgesia (PCA) up to 24 hours postoperatively. 22, 23, 24 Avoiding the drains: Drains can affect patient's ability to mobilise easily and can, therefore, raise a psychological barrier to patient's active participation in their rehabilitation. Surgical drains have not been shown to reduce complications and can actually cause problems such as infection. Some believe that there may be occasional clinical indication for using drains. 3

Regular postoperative analgesia: Suggested analgesia post operatively is regular paracetamol and a nonsteroidal anti-inflammatory agent (depending on patient medical history) administered regularly, despite patient appearing pain free. Additional analgesia for the break through pain in the form of tramadol can be given. Pain is often a barrier for early mobilisation. The aim is for the patients to be reporting a pain score of less than 2 on movements.

Minimising the risk of post-operative nausea and vomiting (PONV): PONV can be more stressful than pain and can come in the way of early mobilisation. Appropriate regular anti emetic should be prescribed routinely so is to be given at the first indication of symptoms. In our department, we have a low threshold of using ondansetron for prevention/ treatment of PONV. Early enteral nutrition and optimal fluid management: $(3,6)$ Early oral or enteral feeding is associated with an improved clinical outcome and it 
has been shown to be safe and well tolerated. It is dependent on using appropriate anaesthesia and analgesia, nausea and vomiting prophylaxis and optimal fluid balance.

Suboptimal fluid balance can impair the wound healing, affect tissue oxygenation, leading to prolonged hospitalisation. The best way to limit postoperative intravenous fluid administration is to stop intravenous infusion and early return to oral or enteral fluid therapy.

Early mobilisation: Early mobilisation maintains the muscle mass, promotes muscle strength and reduces the respiratory complication. It also helps prevent development of deep vein thrombosis. The aim is to mobilise the patient out of bed on the day of surgery. At 2-3 hours postoperative time on return to the ward, the patients are encouraged to sit in the bed. If they feel better, they are encouraged to stand with Zimmer frame with the help of physiotherapist and nurse. Once they feel confident, they can walk to the toilet on the same day of operation. This gives confidence, sense of well-being to the patient and motivation for mobilisation from the next day onwards.

\section{Evidence for ERAS protocols in surgery is available from colorectal resections:}

ERAS protocols have been shown to be associated with faster recovery and reduced length of stay in hospital compared with traditional colorectal resections. ERAS protocols were associated with 2.45 days shorter primary hospital stay and 2.46 days shorter total hospital stay. Morbidity was lower in the ERAS group. 6

\section{Materials and method:}

ERAS programme was introduced as a structured protocol in the department of orthopaedics in Princess of Wales Hospital, ABM University Health Board, in July 2011. This comprised of;

1. Pre-operative education and assessment of all the patients, due to undergo hip and knee replacement, in the preadmission clinic lead by the consultant orthopaedic surgeon (KS) and consultant anaesthetist along with the arthroplasty nurse practitioner, physiotherapist, occupational therapist, and arthroplasty ward nurse 6 weeks before the planned surgery. The patients are given information booklet for the new approach, what it involves and "what to expect".

2. Minimal perioperative starving with preoperative carbohydrate loading at 12 and 2-3 hours before the surgery. All the patients are given 2 sachets of "preload" (a neutral tasting carbohydrate loading drink) on the day of preassessment; each sachet contains 50 grams of carbohydrate preload powder to be mixed with $400 \mathrm{mls}$ of water and to be drunk over 15 minutes.

3. No routine use of the drains.

4. Routine thromboprophylaxis with an oral anticoagulant agent.

5. Multimodal analgesia

6. Local anaesthetic infiltration intraoperatively: (Solution made of $30 \mathrm{mls}$ of $0.5 \%$ Bupivacaine, $70 \mathrm{mls}$ of saline and $1 \mathrm{mls}$ of 1:1000 adrenaline)

7. Regular oral non-narcotic analgesia and anti-emetic with minimal use of the morphinoid drugs.

8. Structured early- on the day of surgery- postoperative weight bearing mobilisation programme.

9. Early oral feeding

10. Early discharge on day 3-4 postoperative whenever possible.

11. All the discharged patients are contacted over telephone at 48 hours post discharge by an arthroplasty nurse practitioner about their general condition and whether they have any concerns. 
We have compared the length of inpatient stay, day of mobilisation, postoperative blood transfusion and adverse outcome for the patients undergoing hip or knee replacement by a single surgeon (KS) over a period of two years. All the patients were given a diary to fill the questionnaire during their stay and they were collected on the day of discharge. The diary asked the patient about the severity of pain, nausea, vomiting, day of weight bearing mobilisation, overall satisfaction. The pain has been graded from 0 to 3 , with 0 as no pain and 3 as severe pain.

The data has been collected prospectively for the ERAS Patients, from July 2011 to June 2012. we compared the results with the patients who underwent hip or knee arthroplasty in a preceding year, (NON ERAS) from July 2010 to June 2011 retrospectively.

\section{Results}

In the ERAS group, a total 138 patients underwent hip or knee replacement, hip resurfacing arthroplasty or oxford uni-compartmental arthroplasty from July 2011 to June 2012 performed by single surgeon (KS). 49 patients underwent total knee replacement, 44 total hip replacements while 31 underwent hip resurfacing. 2 patients underwent Oxford Uni-compartmental knee replacement and 3 underwent revision hip replacement. 9 patients underwent simultaneous bilateral knee replacement.

In the Non ERAS group, 140 patients underwent hip or knee arthroplasty, resurfacing or oxford unicompartmental knee replacement in the previous year by the same surgeon (KS). 63 patients underwent knee replacement, 41 hip replacement, 35 hip resurfacing and one patient underwent oxford unicompartmental knee replacement.

Average hospital in patient stay for the ERAS patients was 4.12 days with $45.5 \%$ of the patients having stayed less than or equal to 3 days. $73.10 \%$ of the patients had inpatient hospital stay of less than or equal to 5 days. Average stay for TKR (total knee replacement) patients was 4.08 days, while bilateral TKR patients had an average stay of 4.8 days. The average hospital inpatient stay for THR patients was 5.4 days. The patients who underwent resurfacing had an average hospital inpatient stay of 3.7 days.

In the non-ERAS group, the average stay of patients was 8.34 days with only $7.75 \%$ of the patients being discharged in less than or equal to 3 days, and $24.08 \%$ of the patients being discharged in less than or equal to 5 days. Average hospital inpatient stay for the patients who underwent TKR was 7.9 days without ERAS protocol, while the patients who underwent hip replacement had an average hospital inpatient stay of 8.36 days. The patients who underwent hip resurfacing without ERAS protocol had an average hospital inpatient stay of 6.17 days.

None of the patient under ERAS protocol was prescribed PCA (Patient controlled Analgesia-Morphine) routinely. They were prescribed regular paracetamol along with anti-inflammatory (where it is not contraindicated) along with pregabalin. $55.6 \%$ of the patients felt that they had adequate pain relief postoperatively, while $44.4 \%$ needed top up pain relief in terms of morphine. $78 \%$ of the patients in ERAS protocol had not experienced nausea or vomiting postoperatively, while $14 \%$ suffered only nausea and $4 \%$ had vomiting. $4 \%$ of the patients did not comment on their diary. The nausea, vomiting and pain scores are not available for the NON ERAS group of patients. 42 patients out of 98 who completed the diary walked on the day of surgery to the toilet full weight bearing, while none of the patients walked on the day of surgery in the NON ERAS group. None of the patients on the ERAS protocol required 
postoperative blood transfusion, while 14 out of 140 patients required blood transfusion after the elective joint replacement surgery in NON ERAS group.

All the patients (100\%) were very satisfied with the overall management starting from the day of preassessment clinic. 2 patients on the ERAS protocol developed DVT -both TKR- one occlusive below knee DVT and one non occlusive below knee DVT. 3 patients developed below knee DVT in a NON ERAS group of patients, and one developed PE. 2 patients on the ERAS protocol developed wound problems, one with wound oozing and second patient had haematoma. Both settled down with conservative management. 5 of the NON ERAS group had wound oozing problem, which also settled with conservative management. There was no incidence of postoperative wound infection in both the groups. 2 of the ERAS group patient dislocated their hips (after primary THR) following fall. One patient's hip became stable after closed manipulation and abduction bracing for 6 weeks, while the second patient required revision surgery to augment the cup orientation. One patient on the ERAS protocol developed aspiration pneumonia following general anaesthetic for which he was treated in the intensive care for 3 days. He recovered completely.

\section{Discussion and conclusion:}

The comparison data shows significant decrease in the hospital in patient stay for the patients in the ERAS protocol as compared with the standard NON ERAS group of patients. The pain was better controlled and nearly $50 \%$ of the patients who have completed diary have mobilised full weight bearing postoperatively on the day of surgery. Earlier mobilisation on the day of surgery makes the impact of surgery psychologically less stressful and also imparts the sense of well-being. Having walked on the weekday of surgery gives a confidence boost to the patients and they are more likely to be out of bed mobilising for next few days making early recovery, avoiding complication of bed rest and likely to go home earlier.

On average the patients undergoing hip resurfacing are much younger than the patients undergoing standard hip or knee replacement surgery and they have fewer or no associated medical co morbidity. This accounts for the faster recovery and shorter hospital stay for the patient after the surgery, which is evident on both, ERAS and NON ERAS, groups of patients. The overall patient experience of undergoing a major surgery was satisfactory with no increased risk of complications.

The current evidence and our study shows that the implementation of the ERAS protocol in hip and knee replacement surgery is associated with improved patient experience, faster recovery and shorter hospital in patient stay with no increase in complication. This will also result in reduced risk of hospital acquired infection, increasing patient's confidence in the organisation.

Shorter hospital stay will free up much needed hospital beds, including ITU/HDU, where applicable, with a potential to treat more patients with the same resources leading to increased capacity for the trust and longer term tariff benefits.

\section{References:}

1. Kehlet, $\mathrm{H}$ and Morgensen T. 1999, 'Hospital stay of 2 days after open sigmoidectomy with a multimodal rehabilitation programme British Journal of Surgery, Feb; 86 (2):227-30.

2. Modernising Care for Patients undergoing Major Surgery: www. reducinglengthofstay.org.

3. 1000 Lives Plus: www.1000lives.wales.nhs.uk

4. Urbach, DR and Baxter, NN. 2005. Reducing variation in surgical care. BMJ 2005; 330 :1401 doi: 10.1136/bmj.330.7505.1401 (Published 16 June 2005) 
5. Delivering Enhanced Recovery after Surgery: Helping patients recover better after surgery. http://tinyurl.com/y2qeplm (Accessed 26th June 2010)

6. Enhanced recovery after surgery programs hastens recovery after colorectal resections. Ned Abraham, Sinan Albayati. world journal of Gastrointestinal Surgery 2011 January 27; 3(1):1-6, (editorial)

7. National joint registry for England and wales : 7th annual report. 2010

8. Scott Beattie w, et al (2009), 'Risk Associated with Preoperative Anaemia in Noncardiac Surgery', Anaesthesiology; 110:574-81

9. AAGBI Safety Guideline (2010), 'Pre-operative assessment and patient preparation, the role of the anaesthetist', The Association of Anaesthetists of Great Britain and Ireland

10. Oxford textbook of orthopaedics and trauma 2002

11. http://hospital.blood.co.uk/library/pdf/INF_PCS_HL_011_01_Iron_leaflet.pdf

12. www.transfusionguidelines.org.uk

13. www.nhs.uk/Conditions/Anaemia-iron-deficiency/Pages/MapofMedicinepage.aspx

14. UK Prospective Diabetes Study, Diabetes Control and Complications Trial

15. www.lho.org.uk/viewResource. aspx?id=9776

16. Shourie S. et al (2007), 'Pre-operative screening for excessive alcohol consumption among patients scheduled for elective surgery', Drug and Alcohol Review, 26: 119-125

17. Dix P and Howell S (2001), 'Survey of cancellation rate of hypertensive patients undergoing anaesthesia and elective surgery, British'. Journal of Anaesthesia, 86 (6):789-793

18. Goldman L and Caldera DL (1979), 'Risks of general anaesthesia and elective surgery in the hypertensive patient', Anaesthesiology; 50: 285-92

19. Ramsay LE, Williams B, Johnston GD, et al (1999), 'British Hypertension Society guidelines for hypertension management', summary. BMJ; 319: 630-5

20. 'The Health Promotion Action plan for Older People in wales' (2004), Health Challenge wales, welsh Government

21. Welsh Risk Pool (2005), 'welsh risk management standards 2004-2005', St. Asaph:

22. Local infiltration analgesia: a technique for the control of acute postoperative pain following knee and hip surgery. A case study of 325 patients. Dennis R Kerr, Lawrence Kohan: Acta orthopaedic 2008; 79 (2): 174-183

23. Efficacy of periarticular multimodal drug injection in total knee arthroplasty. A Randomised trial: Constant A. Busch, Benjamin J. Shore, Rakesh Bhandari, Su Ganapathi, Steven J. MacDonald, Robert B. Bourne, Cecil H. Rorabeck and Richard w. McCalden.: JBJS Am. 88:959-963,2006. Doi:10.2106/JBJS.E.00344

24. Pain control after total knee arthroplasty: A Prospective study comparing local infiltration anaesthesia and epidural anaesthesia: Martin Thorsell, MD; Petter Hoist, MD: Hans Christian Hyldahl, MD: Lars Weidenhielm, MD: Orthopaedics 33 w, 75-80 (Feb 2010)

25. Anti-Fibrinolytic use for minimising perioperative allogenic blood transfusion (review) 2007: Henry DA, Carsen PA et al: Cochrane collaboration

26. Magnesium as an adjuvant to postoperative analgesia: A systematic review of randomised trials: Christopher Lysakowski, MD: Anesth Analg 2007: 104: 1532-9

27. Gabapentin and postoperative pain: a qualitative and quantitative systematic review, with focus on procedure: Ole Mathiesen, Steen Moiniche and Jorgen B Dahl: BMC Anesthesiology 2007, 7:6

28. Pregabalin and Dexamethasone improves post-operative pain treatment after tonsillectomy: $O$ Mathiesen, D G Jorgensen et al: Acta Anaesthesiol Scand 2011; 55:297-305 
29. Single dose Dexamethasone reduces dynamic pain after total hip arthroplasty: Anaesth Analg: 2008: 106:1253-7

30. Multimodal analgesia with Gabapentin, ketamine and Dexamethasone in combination with paracetamol and ketorolac after hip arthroplasty : a preliminary study: Michael L. Rasmussen, Ole Mathiesen, Gerd Dierking et al: Eur J Anaeshtesiol 2010;27: 324-330

31. Low risk of thromboembolic complications after fast tract hip and knee arthroplasty. : Henrik Husted, Kristian Stahl Otte et al: Acta Orthopaedica 2010; 81 (5): 599-605 